\title{
BJGP OPEN
}

\section{The impact of remote consultations on brief conversations in general practice}

\author{
Mughal, Faraz; Atherton, Helen; Awan, Hassan; Kingstone, Tom; Poppleton, \\ Aaron; Silverwood, Victoria; Chew-Graham, Carolyn A.
}

DOI: https://doi.org/10.3399/BJGPO.2021.0199

To access the most recent version of this article, please click the DOI URL in the line above.

Received 19 October 2021

Revised 24 December 2021

Accepted 22 Febuary 2022

(C) 2022 The Author(s). This is an Open Access article distributed under the terms of the Creative Commons Attribution 4.0 License (http://creativecommons.org/licenses/by/4.0/). Published by BJGP Open. For editorial process and policies, see: https://bjgpopen.org/authors/bjgp-open-editorial-process-and-policies

When citing this article please include the DOI provided above.

\section{Author Accepted Manuscript}

This is an 'author accepted manuscript': a manuscript that has been accepted for publication in BJGP Open, but which has not yet undergone subediting, typesetting, or correction. Errors discovered and corrected during this process may materially alter the content of this manuscript, and the latest published version (the Version of Record) should be used in preference to any preceding versions 


\section{Title page}

Research call: telehealth

Manuscript name: the impact of remote consultations on brief conversations in general practice

Manuscript type: commentary

Authors: Mughal F, Atherton H, Awan H, Kingstone T, Poppleton A, Silverwood V, ChewGraham CA

Corresponding author: Dr Faraz Mughal, DCH, MPhil, FRCGP, GP and NIHR Doctoral Fellow, School of Medicine, Keele University, Keele, ST5 5BG, UK. F.mughal@keele.ac.uk

Unit of Academic Primary Care, Warwick Medical School, University of Warwick, Coventry, CV4 7AL.

Associate Professor Helen Atherton, MSc, MPH, PhD, Unit of Academic Primary Care, Warwick Medical School, University of Warwick

Dr Hassan Awan, MRCGP, MSc, PGCME, FHEA, School of Medicine, Keele University, Keele, ST5 5BG, UK,

Dr Tom Kingstone, MRes, PhD, School of Medicine, Keele University, ST5 5BG, Keele, UK,

Dr Aaron Poppleton, MRCGP, MSc, MPH, School of Medicine, MPH, MRCGP Keele University, Keele, ST5 5BG, UK,

Dr Victoria Silverwood, PGCert, PGDip, MRCGP, MPhil, School of Medicine, Keele University, Keele, ST5 5BG, UK,

Professor Carolyn A Chew-Graham, MD, FRCGP, School of Medicine, Keele University, Keele, ST5 5BG, UK, 


\section{Background}

The consultation between the family doctor and patient is the cornerstone of general practice, supporting relationship-based care and continuity, which can improve patient outcomes. $(1,2)$ It enables rapport to be established and provides a setting to begin conversations on sensitive topics such as weight management in a patient with osteoarthritis, or alcohol consumption in someone with elevated liver function tests. Brief conversations in general practice are introductory enquiries or exchanges that are initiated by the general practitioner (GP), often as a stepping stone to more detailed discussions, to improve patient's capability to make positive changes for their health and wellbeing.

Clinical encounter with family

FM recollects a face-to-face consultation in 2016 as a GP, where after handling the initial presenting problem of the child, he spoke to the parents about the child's diet. He discovered that the parents provided the child with fast food (chicken and chips) each day for dinner after school. The parents were not aware that this was detrimental to their child's health. FM used this disclosure to provide brief advice on the importance of a balanced diet and offered some low-cost easy to prepare alternatives.

Dealing with and managing the patient's presenting complaint is important, but using the opportunity to explore aspects of health promotion, prevention, and behaviour change is key to realising the exceptional potential of the primary care consultation.(3) Leaning on the 'Making Every Contact Count' public health initiative, GPs can use consultations to initiate brief conversations, which in turn can lead to the delivery of interventions and potentially result in long lasting benefits for patients and the NHS.(4, 5)

In a consultation with someone who is experiencing distress, GPs who utilise an open questioning style and sensitively ask about self-harm, can facilitate further discussion around self-harm and suicide and this can be life-saving.(6) In the management of obesity, language is important and seeking the permission of the patient: 'would you mind if we spoke about your weight?' for example, provides a chance for patients to share concerns or ask questions.(7) Patients perceive receiving a brief health behaviour change intervention as appropriate and helpful, especially in long term conditions, with the doctor-patient relationship the foundation of the conversation.(8)

In this commentary we discuss how the increased reliance on remote consulting, exacerbated by COVID-19, may impact on the opportunity for brief conversations in general practice globally; and outline implications for clinical practice and policy, and suggestions for future research.

\section{Remote consulting and COVID-19}

The delivery of general practice consultations across countries has changed substantially because of COVID-19. $(9,10)$ Many countries increased their use of remote consultations (telephone, video, email, and other forms of internet communication) to continue to provide essential primary care services while mitigating the risk of COVID19 in staff and patients.(10) In England across one clinical commissioning group there 
was a 58\% increase in telephone general practice consultations in early COVID-19 (April 2020) compared to 2019: video and e-consultations were noted to be $1 \%$ or less of consultations.(11) For many primary care clinicians this required on the job learning and necessary engagement with new technologies as they came to rely on these alternative approaches in a short space of time.(12)

\section{Communication in remote consultations}

Different communication mediums have different strengths and weaknesses as modes of consultation. Telephone consultations are acceptable to both GPs and patients and seen as an appropriate consultation mode for non-complex single concerns, including new acute problems, where patients may be asked to attend later for a face-to-face consult.(13) The subtle features noticeable during a face-to-face consultation such as observing body language (sitting uncomfortably or looking away) and non-verbal cues (a wince or a facial reaction) are however lost in a telephone consultation.(14) Patients who perceive they don't communicate well described a lack of trust in telephone consultations and preferred to see a doctor face-to-face where they may be able to better articulate their problems.(15)

Video consultations result in varied experiences for patients reflecting the complexity of using these in practice. Research has shown that patients can encounter difficulty in establishing a doctor-patient relationship using video, feel rushed, and have trouble with turn taking.(16) Conversely, when used as a method of follow up in the context of an existing doctor-patient relationship, patients appreciate the visual element of a video consultation over and above what is achievable in a telephone consultation.(17)

A recent systematic review found that primary care clinicians described concerns with e-consultations (email or online messaging) around the potential negative effect on the doctor-patient relationship due to the need for GPs to use non-technical language; however, the ability to communicate immediately and in some patients, their access to notes, enhanced trust and communication, and strengthened the therapeutic relationship.(18)

These findings demonstrate it is crucial to develop doctor-patient rapport when using a new medium and this in turn can enable brief conversations to occur. If FM undertook a telephone or e-consultation in the above consultation, he would not have seen the child's size or perhaps the parents would not have felt comfortable talking about food habits, and therefore the opportunity for the brief conversation may have been lost.

\section{Missed conversations are missed opportunities}

To maximise the opportunity for brief conversations, establishing GP-patient rapport in remote consultations is crucial. Missed brief conversations are missed chances for promoting good health and well-being in general practice. In the long term, losing the opportunity for brief conversations may impact on patient populations, especially in those with long-term conditions where the potential for positive change may be greatest, and subsequently negatively burden primary care services and systems. This requires ongoing evaluation as remote consulting becomes more established in general practice. 


\section{Implications for practice, policy, and future research}

The capability of clinicians to adopt and confidently use remote consultation methods, which most GPs have not trained in, cannot be assumed and evidence-informed training will need upscaling. In addition, GPs need to be aware that consulting remotely may affect rapport-building and consider this when initiating brief conversations. Policymakers need to contemplate the growing evidence on relationship-based care and ensure this is protected and encouraged in a COVID-19 context with growing time constraints: this should be prioritised by primary care systems worldwide.

Retaining relationship-based care at the heart of the general practice consultation will enable brief conversations to continue for the benefit of patients, the public, clinicians, and health services. Future research should prioritise how the range of remote consultation options can support patients in their ongoing care. Conversation analyses across different modes of remote consultations would enable an in-depth examination of how brief conversations occur. The views of patients and clinicians on remote brief conversations need to be explored to establish what elements would be acceptable and useful, therefore informing best practice recommendations for general practice.

Funding: FM is funded by a NIHR Doctoral Fellowship (NIHR300957). Hassan Awan, VS, and AP are funded by Wellcome Clinical Doctoral Fellowships. CCG is part-funded by the NIHR West Midlands Applied Research Collaboration. Helen Atherton is part funded by Research Council of Norway and NIHR grants: NIHR128268 and NIHR202614. The views expressed in this publication are those of the authors, and not necessarily those of the NHS, NIHR, Wellcome Trust, or the Department of Health and Social Care.

Competing interests: Helen A spoke on relationship-based care at RCGP Annual Conference 2021, and is a member of the Evaluation Steering Committee, Digital First Primary Care, NHS England. 
1. Baker R, Freeman GK, Haggerty JL, et al. Primary medical care continuity and patient mortality: a systematic review. Br J Gen Pract. 2020;70(698):e600-e11.

2. Royal College of General Practitioners. The power of relationships: what is relationship-based care and why is it important? General practice COVID-19 recovery. 2021. 3. Stott NC, Davis RH. The exceptional potential in each primary care consultation. J R Coll Gen Pract. 1979;29(201):201-5.

4. NHS Health Education England. Making Every Contact Count [Available from: https://www.makingeverycontactcount.co.uk/ Accessed 23 December 2021].

5. Aveyard $P$, Lewis $A$, Tearne $S$, et al. Screening and brief intervention for obesity in primary care: a parallel, two-arm, randomised trial. Lancet. 2016;388(10059):2492-500.

6. Ford J, Thomas F, Byng R, McCabe R. Asking about self-harm and suicide in primary care: Moral and practical dimensions. Patient Educ Couns. 2021;104(4):826-35.

7. Obesity UK and Obesity4Thought. Language matters: obesity. 2020

8. Keyworth C, Epton T, Goldthorpe J, et al. Perceptions of receiving behaviour change interventions from GPs during routine consultations: A qualitative study. PLoS One. 2020;15(5):e0233399.

9. Huston P, Campbell J, Russell G, et al. COVID-19 and primary care in six countries. BJGP Open. 2020;4(4).

10. Haldane $\mathrm{V}$, Zhang Z, Abbas RF, et al. National primary care responses to COVID-19: a rapid review of the literature. BMJ Open. 2020;10(12):e041622.

11. Murphy M, Scott LJ, Salisbury C, et al. Implementation of remote consulting in UK primary care following the COVID-19 pandemic: a mixed-methods longitudinal study. $\mathrm{Br} \mathrm{J}$ Gen Pract. 2021;71(704):e166-e77.

12. Marshall M, Howe A, Howsam G, et al. COVID-19: a danger and an opportunity for the future of general practice. Br J Gen Pract. 2020;70(695):270-1.

13. Hewitt $\mathrm{H}$, Gafaranga J, McKinstry B. Comparison of face-to-face and telephone consultations in primary care: qualitative analysis. Br J Gen Pract. 2010;60(574):e201-12.

14. McKinstry B, Watson P, Pinnock $\mathrm{H}$, et al. Telephone consulting in primary care: a triangulated qualitative study of patients and providers. Br J Gen Pract. 2009;59(563):e20918.

15. Pope C, McKenna G, Turnbull J, et al. Navigating and making sense of urgent and emergency care processes and provision. Health Expect. 2019;22(3):435-43.

16. Gordon HS, Solanki P, Bokhour BG, Gopal RK. "I'm Not Feeling Like I'm Part of the Conversation" Patients' Perspectives on Communicating in Clinical Video Telehealth Visits. J Gen Intern Med. 2020;35(6):1751-8.

17. Donaghy $\mathrm{E}$, Atherton $\mathrm{H}$, Hammersley $\mathrm{V}$, et al. Acceptability, benefits, and challenges of video consulting: a qualitative study in primary care. Br J Gen Pract. 2019;69(686):e586e94.

18. Mold F, Hendy J, Lai YL, de Lusignan S. Electronic Consultation in Primary Care Between Providers and Patients: Systematic Review. JMIR Med Inform. 2019;7(4):e13042. 